\title{
Measurement of Sub Degree Angular Carbon Fiber Tow Misalignment
}

\author{
William C. Wilson ${ }^{1}$, Jason P. Moore ${ }^{1}$, Hunter $\mathrm{McCraw}^{2}$ \\ ${ }^{1}$ NASA Langley Research Center, Hampton, VA, USA \\ ${ }^{2}$ Embry-Riddle Aeronautical University, Prescott, AZ, USA
}

\begin{abstract}
NASA is investigating the use of carbon fiber tow steering to tune aeroelastic characteristics in advanced composite structures. In support of that effort, NASA is also investigating methods of measuring the angle of carbon fiber tows as they are placed. This work presents the results of using microwave reflectometry in the $\sim 2 \mathrm{GHz}$ region to measure carbon fiber tow angles at $0.1^{\circ}$ resolution.
\end{abstract}

Index Terms - Microwave; frequency domain reflectometry; electromagnetic; carbon fiber; tape orientation.

\section{INTRODUCTION}

Motivated by a desire to reduce drag, weight, and fuel consumption of aircraft, NASA continues to investigate new wing structures assembled of advanced materials such as carbon fiber reinforced polymer (CFRP). Because a side effect of reducing structural weight is an increased susceptibility of wings to enter into flutter conditions, methods of reducing the onset of flutter are concurrently being explored. One method of reducing the probability of flutter onset in a CFRP wing structure is to aeroelastically "tune" the structural characteristics via carbon fiber tow steering $[1,2]$. Both flutter and gust response can be modified using tow steering methods [3]. In addition to modifying aeroelastic properties, the continuous tow steering technique also has the advantage of reducing the design weight by $34 \%$ to $38 \%$ when compared to conventional straight fiber methods [4]. Structural performance improvements, such as increased buckling load limits, can also be optimized by tow steering [5]. To improve the angular accuracy of tow steered layups, microwaves are being investigated as a means to remotely determine the angle of the carbon fiber tow with the ultimate goal of creating a remote non-contact method of monitoring the angle of the tow during the layup process.

Previous work has demonstrated that a resolution of $1^{\circ}$ can be achieved in the far field using microwaves [6]. In this prior research, frequencies in the 500-1000 $\mathrm{MHz}$ range were used because they offered the desirable characteristics of low noise and pronounced electro-magnetic (EM) signature frequency shifts versus angular changes in a carbon fiber tow. This new work is motivated by the desire to implement the technique on an automated fiber placement system such as that shown in Fig. 1.

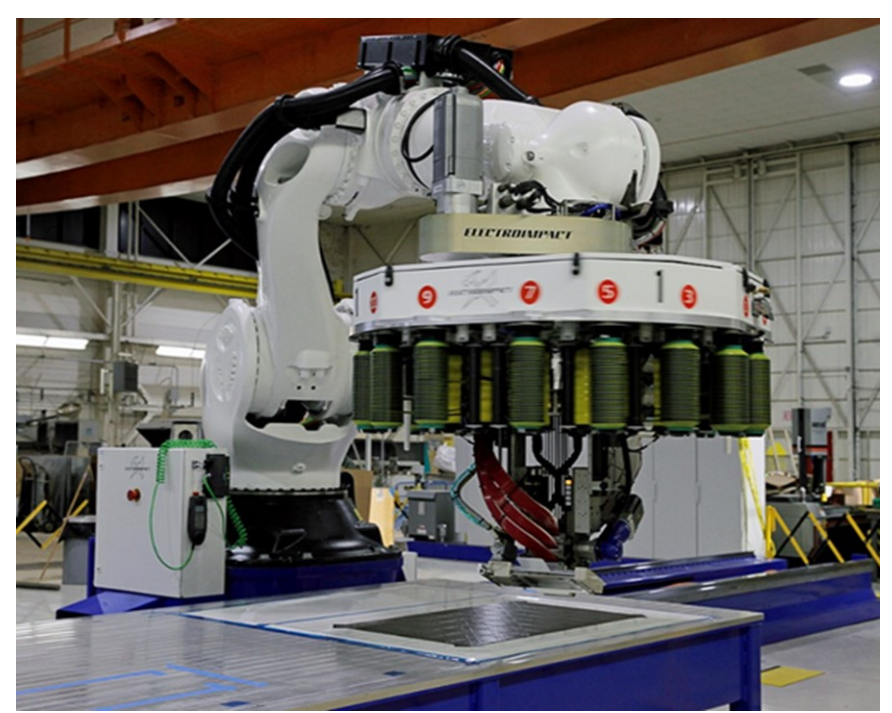

Fig. 1. NASA Langley Research Center's carbon fiber fabrication robot ISAAC (Integrated Structural Assembly of Advanced Composites).

Presently, the largest in-situ component of the sensing equipment is the antenna; the size of which depends on its operational frequency range and gain. Specifically, larger wavelength EM fields require larger antennas to produce them. An additional need is the ability to measure sub-degree tow angle deviation of the layup. Addressing those two requirements, the use of higher frequency EM radiation in the $\sim 2 \mathrm{GHz}$ region to measure carbon fiber tow angular deviations less than $1^{\circ}$ is presented.

\section{EXPERIMENTAL SETUP}

The experimental setup consists of a microwave reflectometer positioned to record the EM signature of a 6.4 $\mathrm{mm}$ wide, $250 \mathrm{~mm}$ long IM7 carbon fiber tape. The EM signature of the carbon fiber tape was interrogated using a vector network analyzer connected to a 2-4 GHz, linearly polarized, octave horn antenna with an aperture of $124 \mathrm{~mm}$ by $164 \mathrm{~mm}$. 


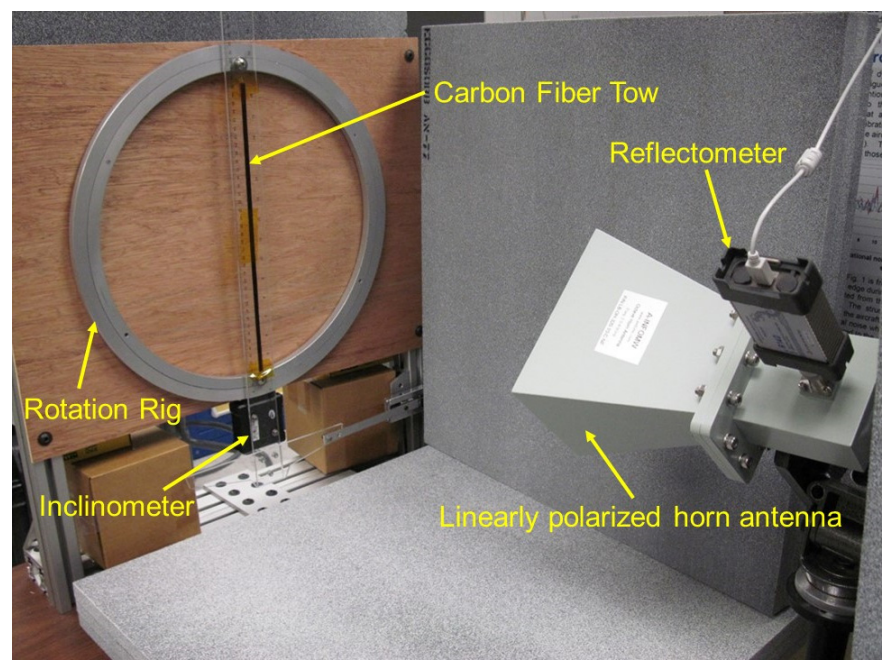

Fig. 2. Experimental setup: carbon fiber tow, tow rotator rig, inclinometer, and linear polarized horn antenna.

This antenna is smaller than in the previous work $(700 \mathrm{MHz}$ - $18 \mathrm{GHz}$, linearly polarized, dual-ridge horn antenna. $142 \mathrm{~mm}$ by $244 \mathrm{~mm}$ aperture). The vector network analyzer (VNA) serves as a single port reflectometer and was connected to a computer where the data was collected and stored. The test rig for positioning the carbon fiber tape was constructed of an aluminum turn table torus with an angle inclinometer placed on a $3.175 \mathrm{~mm}$-thick wood board (Fig. 2). The carbon fiber tape was attached to the rotation ring using Kapton tape. The entire test setup was enclosed in a layer of microwave-absorbing foam to isolate the system and lower external noise/interference.

Because in previous work, the maximum sensitivity to carbon fiber tow orientation was achieved when the antenna transverse magnetic (TM) polarization axis was oriented at either $45^{\circ}$ or $135^{\circ}$ relative to the layout direction [6], the antenna was secured $510 \mathrm{~mm}$ away from the carbon fiber tape with its TM polarization axis aligned to the global $45^{\circ}$ axis of the wood base (Fig. 2). Calculating the far field distance $d_{f}$ using

$$
d_{f}=2\left(D^{2}\right) / \lambda
$$

where $D$ is the largest antenna aperture dimension $(164 \mathrm{~mm}$,) and $\lambda$ is the wavelength $(144.8 \mathrm{~mm}$ at $2.670 \mathrm{GHz})$, yields $d_{f}=$ $371.4 \mathrm{~mm}$. This establishes that the $510 \mathrm{~mm}$ antenna-to-tape distance for this experiment is in the far field.

Before taking data, the basic $\mathrm{S}_{11}$ response (reflection coefficient) of the carbon fiber tape was recorded in the 2.5 $\mathrm{GHz}$ to $3 \mathrm{GHz}$ frequency band (Fig. 3).

This investigation focuses on the frequency shift of the dominant trough located at approximately $2670 \mathrm{MHz}$ (Fig. 3).

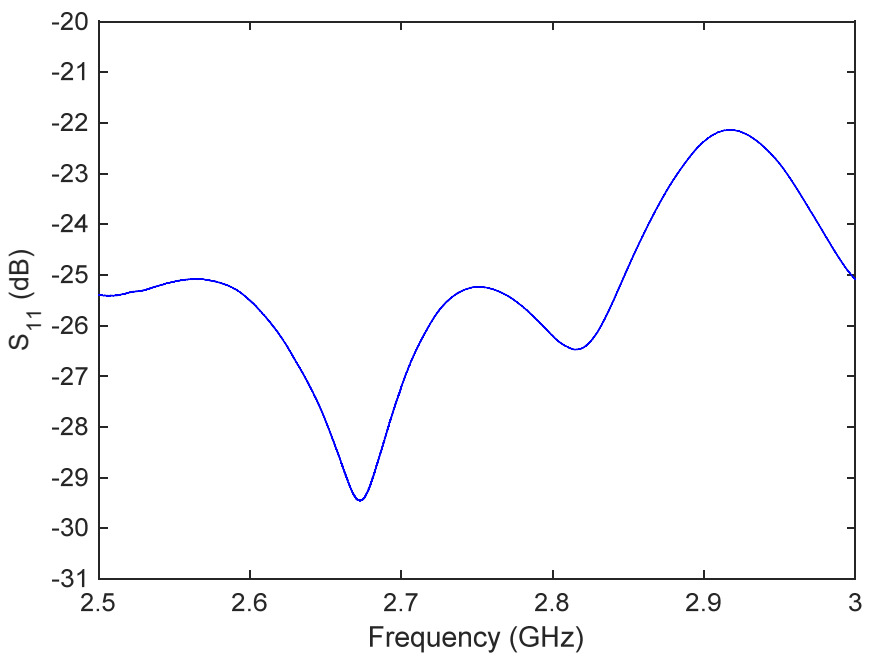

Fig. 3. The EM signature (log magnitude $\mathrm{S}_{11}$ response) of the carbon fiber tape from $2.5 \mathrm{GHz}$ to $3 \mathrm{GHz}$.

The frequency band around the trough can be exploited to determine the orientation of the carbon fibers based on frequency response. The specific frequency band chosen for investigation was $2660 \mathrm{MHz}$ to $2680 \mathrm{MHz}$ (Fig. 4), which is below the frequencies where absorption becomes a dominant factor [7].

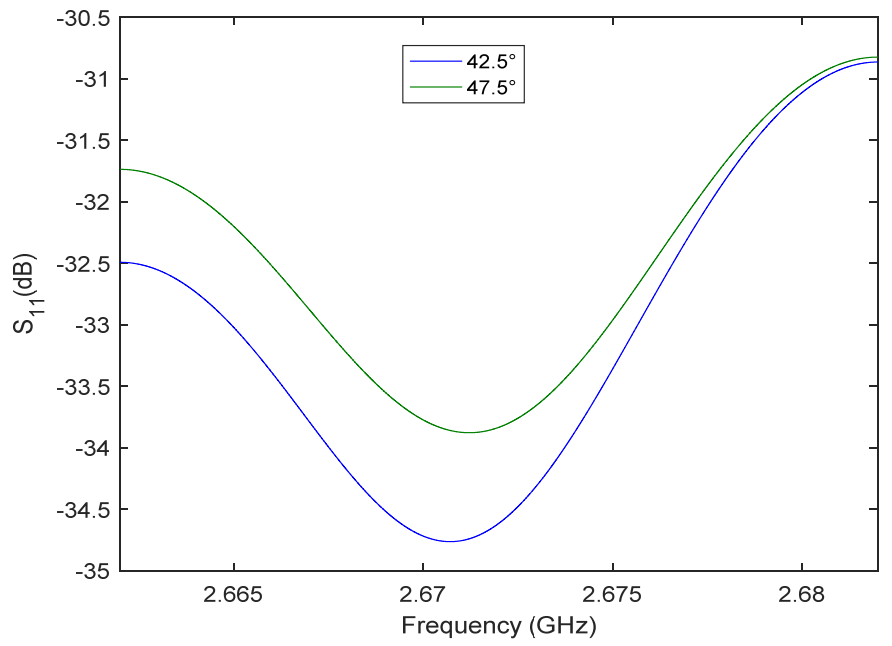

Fig. 4. The EM signature (log magnitude $\mathrm{S}_{11}$ response) of the carbon fiber tape from $2.66 \mathrm{GHz}$ to $2.68 \mathrm{GHz}$, for both $42.5^{\circ}$ and $47.5^{\circ}$.

Time-based gating of the return signal was employed to limit the recorded data to reflections occurring only from a distance of $400 \mathrm{~mm}$ to $600 \mathrm{~mm}$ from the antenna, ensuring that the recorded signal return was from the carbon fiber tape with minimal influence from the surrounding environment. 


\section{RESULTS}

After investigating the EM signature and determining the frequencies of interest, measurements were taken without the carbon fiber tape present on the test rig to ensure that the changes in response were dependent on the carbon fiber tape orientation and not the test rig itself. Without a carbon fiber tape present, the $S_{11}$ and impedance data showed random dependence on the rotation of the test rig from $0^{\circ}$ to $180^{\circ}[6]$.

With the carbon fiber tape in place, the EM signature was recorded across the $2.66 \mathrm{GHz}$ to $2.68 \mathrm{GHz}$ frequency band for the TM-tape orientation range of $42.5^{\circ}$ to $47.5^{\circ}$ at $0.5^{\circ}$ increments. At each angular position, 6 data sets, each consisting of 8001 points across the band of interest, were recorded.

Previous work demonstrated the expected cosine dependence on the angle of the fiber with respect to the polarization direction for $1^{\circ}$ increments [8]. However, within the smaller angular deviation range of this work (below one degree) the impedance dependence on fiber orientation is expected to be nearly linear. Note that due to symmetry we are getting a full cycle in $180^{\circ}$, and therefore the small angle approximation to the cosine applies at $45^{\circ}$ and $135^{\circ}$. Within the 8001 data points in each data set, a search was performed to determine a specific interrogation frequency that would give minimal deviation of the impedance from the expected linear dependence. The frequency of $2.6651 \mathrm{GHz}$ was found to be optimal for making angular measurements using $0.5^{\circ}$ steps. Note that for each run we search for the optimal frequency. Changes in the optimal frequency between runs most likely is due to environmental changes such as temperature.

The EM signature impedance at $2.6651 \mathrm{GHz}$ for each of the 6 data sets at each angular orientation is given in Fig. 5. The changes in impedance as the angle was changed are easily distinguishable in the "stair step" pattern of the data.

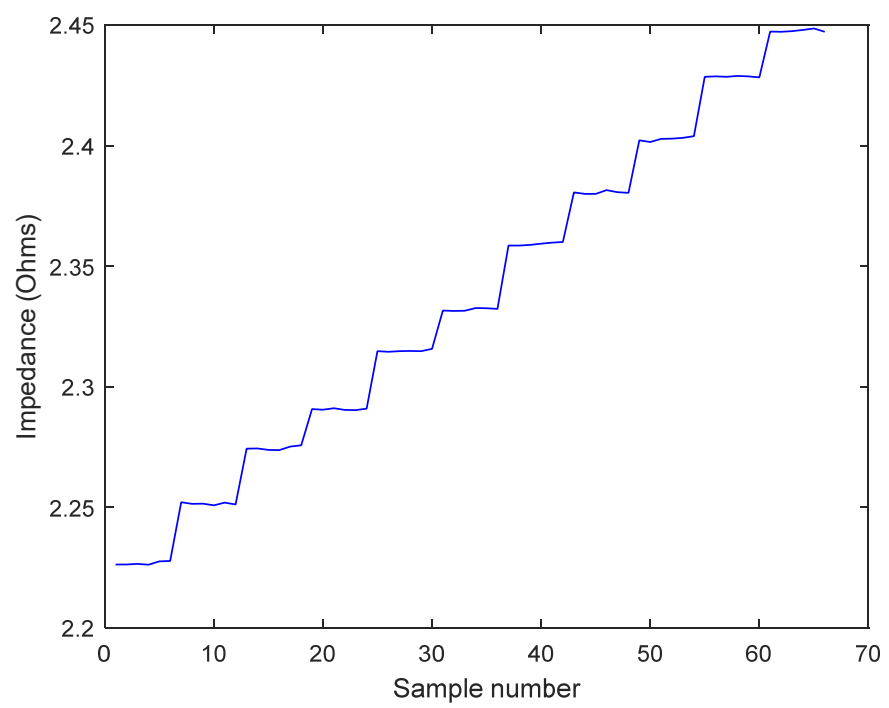

Fig. 5. The EM signature impedance at $2.6651 \mathrm{GHz}$ of 6 sets of 11 angular steps.
To evaluate the linear dependence of impedance on tow angle, the six data points at each individual angle position shown in Fig. 5 were averaged to give a singular impedance value at each angular step (Fig. 6).

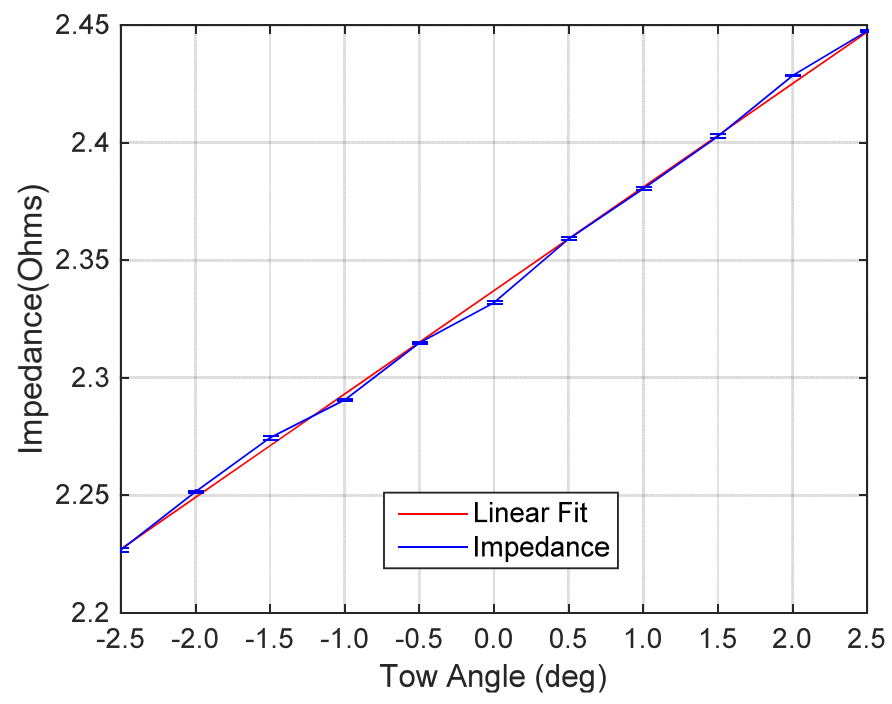

Fig. 6. Change in impedance as a function of tow angle relative to $45^{\circ}$ in angular increments of $0.5^{\circ}$. The error bars indicate one standard deviation.

The expected linear trend in the data of Fig. 6 suggests that sub-degree resolution is attainable. Figs. 7 and 8 show the impedance measurements for the angular tow steps of $0.25^{\circ}$ and $0.1^{\circ}$, respectively, about a $45^{\circ} \mathrm{TM}$-tape orientation.

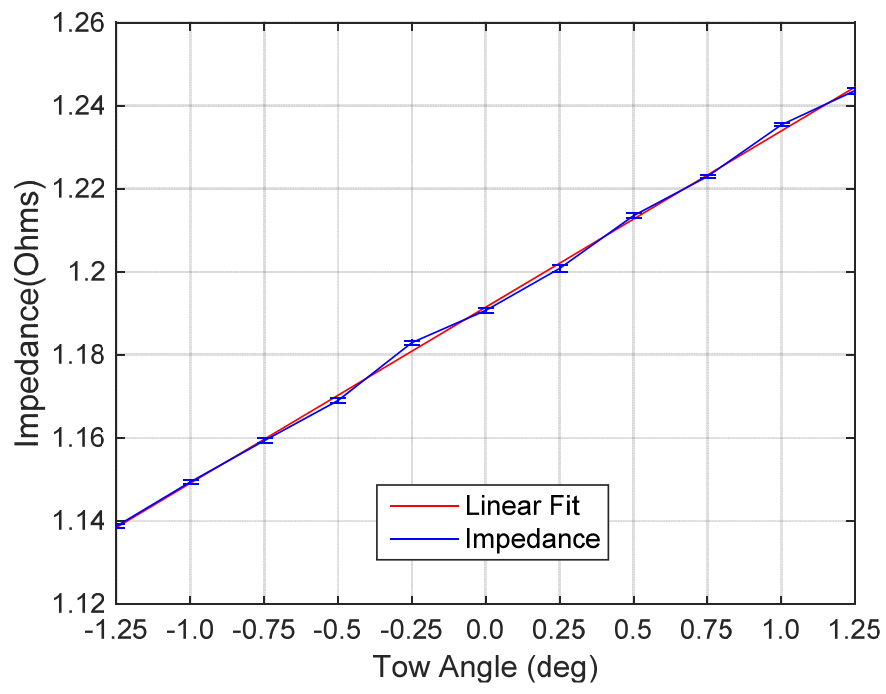

Fig. 7. Change in impedance as a function of tow angle relative to $45^{\circ}$ in angular increments of $0.25^{\circ}$. The error bars indicate one standard deviation. 
At an angular increment of $0.25^{\circ}$ the data trend is still linear, and the deviations from linear are of the same magnitude as those in Fig. 6.

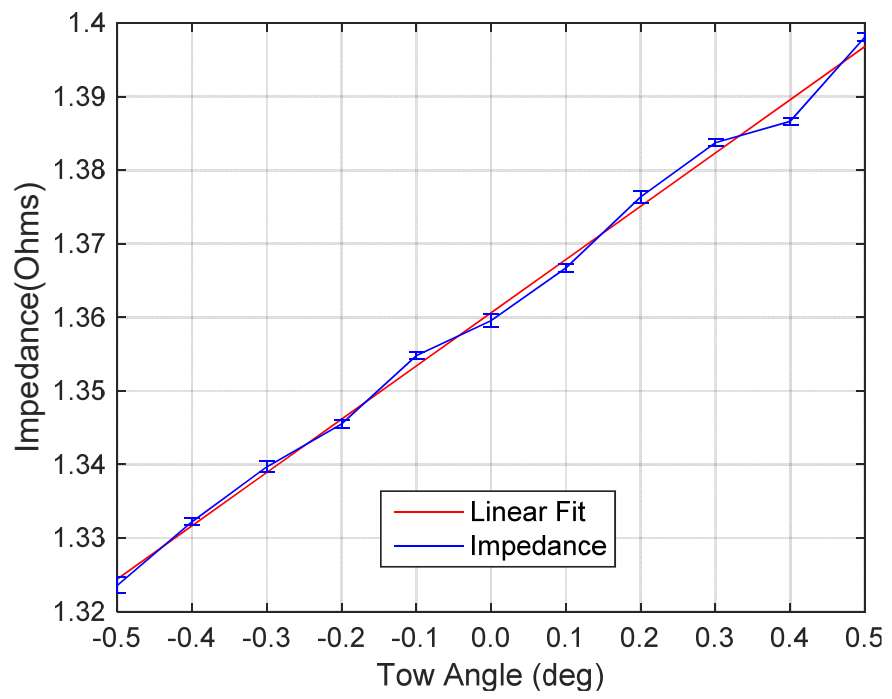

Fig. 8. Change in impedance as a function of tow angle relative to $45^{\circ}$ in angular increments of $0.1^{\circ}$. The error bars indicate one standard deviation.

The data from the $0.1^{\circ}$ increments (Fig. 8) appears dominantly linear and the relatively small error bars strongly suggest that this technique can be used for measuring the angle of carbon fiber tow.

The statistics of the linear fits, specifically the $r^{2}$ values, the lower and upper bounds of the $95 \%$ confidence intervals (CI), and the slopes (sensitivity), are given in Table 1.

TABLE I

STATISTICS AND SENSITIVITY

\begin{tabular}{|c|c|c|c|c|}
\hline Increment & $\boldsymbol{r}^{\mathbf{2}}$ & $\begin{array}{c}\text { Cl lower } \\
\text { bound }\end{array}$ & $\begin{array}{c}\text { Cl upper } \\
\text { bound }\end{array}$ & Slope \\
\hline $0.5^{\circ}$ & 0.999 & 0.042 & 0.045 & 0.044 \\
\hline $0.25^{\circ}$ & 0.999 & 0.041 & 0.043 & 0.042 \\
\hline $0.1^{\circ}$ & 0.997 & 0.069 & 0.076 & 0.072 \\
\hline
\end{tabular}

The average sensitivity across the three tests was calculated using weighted arithmetic means, where the weights were derived from the confidence bounds. Using this method, the average weighted sensitivity was calculated to be $0.045 \pm 0.001$ $\mathrm{ohm}$ per degree. Note that small fluctuations in impedance are expected as environmental conditions such as temperature and humidity fluctuate in the test environment. Environmental changes, therefore, necessitate a search for the optimal frequency for each run. Future work may be able to characterized the temperature dependence and eliminate the need to search for the optimal frequency.

\section{CONCLUSION}

Microwaves have been used to demonstrate sensing of small angular misalignments $\left(0.1^{\circ}\right)$ of a carbon fiber tape sample using impedance from the $S_{11}$ response. The average weighted sensitivity was calculated to be $0.045 \pm 0.001 \mathrm{ohm}$ per degree for these small angular deviations. The use of a narrow bandwidth $(2 \mathrm{GHz})$, high gain $(>11 \mathrm{~dB})$ antenna yielded substantial improvements versus the use of a wide bandwidth $(\sim 18 \mathrm{GHz})$, $\sim 8 \mathrm{~dB}$ gain antenna of previous work.

Future work will investigate techniques for improving both the precision and the accuracy of the measurements, such as increasing the output power and reducing the noise floor of the reflectometer system. Larger interrogation distances and application implementation are also of interest. Temperature characterization should also be performed to eliminate the search for optimal frequency after each run.

\section{REFERENCES}

[1] T. A. Guimaraes, S. G. Castro, et al., "Panel Flutter Analysis and Optimization of Composite Tow Steered Plates," in Proc. 58th AIAA/ASCE/AHS/ASC Structures, Structural Dynamics, and Materials Conference, Grapevine, TX, AIAA 2017-1118, Jan. 913, 2017, pp. 1-14.

[2] B. K. Stanford, C. V. Jutte, et al., "Aeroelastic Benefits of Tow Steering for Composite Plates," Composite Structures, vol. 118, pp. 416-422, Aug. 152014.

[3] O. Stodieck, J. Cooper, et al., "Aeroelastic Tailoring of a Representative Wing Box Using Tow-Steered Composites," AIAA Journal, vol. 55, no. 4, pp. 1425-1439, April 2017.

[4] T. J. Dodwell, R. Butler, et al., "Optimum Fiber Steering of Composite Plates for Buckling and Manufacturability," AIAA Journal, vol. 54, no. 3, pp. 1139-1142, March 2016.

[5] K. C. Wu, J. D. Turpin, et al., "Structural Characterization of Advanced Composite Tow-Steered Shells with Large Cutouts," in Proc. 56th AIAA/ASCE/AHS/ASC Structures, Structural Dynamics, and Materials Conf., AIAA SciTech Forum, Kissimmee, FL, Jan. 5-9, 2015, pp. AIAA 2015-0966, 1-19.

[6] W. C. Wilson, J. P. Moore, et al., "Far field measurements of small angle carbon fiber misalignments," in Proc. Wireless and Microwave Technology Conference (WAMICON), 2017 IEEE 18th, Cocoa Beach, FL, April 24-25, 2017, pp. 1-4.

[7] J. H. Kaiser, "Reflection and Absorption of Microwaves (2-100 $\mathrm{GHz})$ by Carbon-Fiber-Reinforced Composite Surfaces," Research in Nondestructive Evaluation, vol. 5, no. 4, pp. 275283, 1994.

[8] W. C. Wilson, J. P. Moore, et al., "Carbon fiber TOW angle determination using microwave reflectometry," in Proc. SENSORS, 2016 IEEE, Orlando, FL, Oct. 30- Nov. 2, 2016, pp. 1-3. 